\title{
The Birth and Evolution of Fuzzy Logic *
}

\author{
Lotfi A. Zadeh
}

\author{
Computer Science Division \\ Department of Electrical Engineering and Computer Sciences \\ University of California \\ Berkeley, CA 94720, USA
}

Ambassador Takeso Shimoda, President of the Honda Foundation, Mr. Soichiro Honda,

Ambassador Michael Armacost, Ladies and Gentlemen:

I feel honored to have been chosen as the winner of the 1989 Honda Prize. To me this honor has a special significance. First, because it represents an important recognition of the validity of the ideas which were set forth by me more than two decades ago. And second, because these ideas have found a fertile soil in Japan - a country for which I have a deep admiration and affection. It gives me great pleasure to take this opportunity to pay my tribute to the brilliant Japanese scientists and engineers who have contributed so fundamentally to the development of fuzzy logic and its applications and who have played such an important and enlightened role in the international community of fuzzy mathematicians, scientists and engineers.

Unlike traditional logical systems, fuzzy

* Text of a lecture presented on the occasion of the award of the 1989 Honda Prize, Tokyo, Japan : 1989 年度本田賞受賞記念講演 (1989 年 11 月 17 日, ホテル・ オークラ)より logic is aimed at providing a model for modes of reasoning which are approximate rather than exact. In this perspective, the importance of fuzzy logic derives from the fact that almost all of human resoning - and especially commonsense resoning - is approximate in nature.

The basic ideas underlying fuzzy logic were described in my first paper on fuzzy sets which was published in 1965. This paper and my subsequent papers on fuzzy sets drew a mixed reaction. Some academics and especially the late mathematicians Richard Bellman and Grigori Moisil, greeted my ideas with enthusiasm. For the most part, however, what I encountered was skepticism and, on occasion, downright hostility. Today, twenty-five years later, the controversy surrounding fuzzy logic is still with us, though not to the same degree. The numerous applications of fuzzy logic, especially in Japan, are too visible to be denied. However, there are still some who remain 
unconvinced that fuzzy logic has something important to offer. The Cartesian tradition of respect for what is quantitative and precise, and disdain for what is qualitative and imprecise is too deep-seated to be abandoned without a fight. The basic tenet of this tradition was stated succinctly by Lord Kelvin one of the outstanding intellects of the 19 th century - in 1883. He wrote, "In physical science a first essential step in the direction of learning any subject is to find principles of numerical reckoning and practicable methods for measuring some quality connected with it. I often say that when you can measure what you are speaking about and express it in numbers, you know something about it; but when you cannot measure it, when you cannot express it in numbers, your knowledge is of a meagre and and unsatisfactory kind: it may be the beginning of knowledge but you have scarcely, in your thoughts, advanced to the state of science, whatever the matter may be."

In a similar vein, in commenting on one of my papers in 1972, Professor Richard Kalman wrote "I want to say only a few words about what happens to the application of algebra. As an example, I will use a notion of a fuzzy set mentioned by Prof. Zadeh. Let me say quite categorically that there is no such thing as a fuzzy scientific concept, in my opinion."

"We do talk about fuzzy things but they are not scientific concept. Instead, let us view the development of science as something like the following. You look at a vast mass of facts fuzzy or not - and you would like to make some sense out of it. This is usually done through rising to a higher conceptual level, by working harder than the average person. Some people in the past have discovered certain interesting things, formulated their findings in a non-fuzzy way, and therefore we have progressed in science."

In the same vein, a colleague of mine, Professor William Kahan, commented in 1975, "What Zadeh is saying is the same sort of thing as, Technology got us into this mess and now it can't get us out.' Well, technology did not get us into this mess. Greed and weakness and ambivalence got us into this mess. What we need is more logical thinking, not less. The danger of fuzzy theory is that it will encourage the sort of imprecise thinking that has brought us so much trouble."

Despite the skepticism and hostility, there is, today, a rapidly growing international community of scientists and engineers who are actively engaged in the development of fuzzy logic and its applications. In the forefront of this activity are Japan, The People's Republic of China and the Soviet Union. In Japan, in particular, the ideas of fuzzy logic have found a wide acceptance, as is evidenced by the establishment of LIFE, the Laboratory of International Fuzzy Engineering, which is certain to become the world's premier center for research and development relating to fuzzy logic and its applications. One cannot but admire the work done in Japan and the foresight of a small group of eminent scientists who nurtured the field while it was still in its initial stages of development. I should like to pay my tribute to this group a group which was led by the late Professor Kokichi Tanaka of Osaka University, and Professors T.Terano, K.Asai, M.Sugeno, H. 
Tanaka, M.Mizumoto, M.Mukaidono, K.Hirota and M.Umano. I would like to do so, but time does not allow me to mention the names of outstanding scientists and engineers in Japan who are currently contributing so importantly to the development of fuzzy logic and its applications in a wide variety of fields ranging from train control and resource allocation to medical diagnosis and portfolio management.

What are the basic ideas underlying fuzzy logic? What are its potentialities and limitations? These are some of the issues on which I should like to focus in my lecture.

First, a bit of history. Although I am an electrical engineer by training, I have always been a strong believer in the power of mathematics. To me, it was an article of faith that to almost any problem there is a mathematical solution. Like many other workers in systems analysis, information analysis and control, I was driven by a quest for precision, rigor and mathematical sophistication. There was no doubt in my mind that this was the right path to follow.

However, in the course of writing a book with Professor Charles Desoer on linear system theory, I began to realize that there are many concepts in system theory that do not lend themselves to precise definition. For example, one can give a precise definition of a linear system, a stable system, a time-invariant system, etc. But how can one define what is meant by a decentralized system, a slowly-varying system, a reliable system, etc.?

In trying to formulate such definitions, I began to realize that the problem lay in the Aristotelian framework of classical mathematics - a framework which is intolerant of imprecision and partial truth. In essence, a basic assumption in classical mathematics is that a concept must admit of precise definition which partitions the class of all objects into two classes: (i) those objects which are instances of the concept; and (ii) those which are not, with no borderline cases allowed. For example, a function is either continuous or discontinuous; it cannot be continuous to a degree. Similarly, a matrix is either symmetric or not symmetric; it cannot be somewhat symmetric or more or less symmetric or symmetric to a degree. By the same token, a paper published in a mathematical journal is expected to contain precisely stated definitions, assumption and theorems. Generally, a paper would not be acceptable for publication if its conclusions are stated as assertions which are not unequivocally true.

In sharp contrast to the idealized world of mathematics, our perception of the real world is pervaded by concepts which do not have sharply defined boundaries, e.g., tall, fat, many, most, slowly, old, familiar, relevant, much larger than, kind, etc. A key assumption in fuzzy logic is that the denotations of such concepts are fuzzy sets, that is, classes of objects in which the transition from membership to nonmembership is gradual rather than abrupt. Thus, if $A$ is a fuzzy set in a universe of discourse $U$, then every member of $U$ has a grade of membership in $A$ which is usually taken to be a number between 0 and 1 , with 1 and 0 representing full membership and nonmembership, respectively. This function which associates with each object its grade of mem- 
bership in $A$ is called the membership function of $A$. This function defines $A$ as a fuzzy subset of $U$.

It is important to observe that there is an intimate connection between fuzziness and complexity. Thus, a basic characteristic of the human brain, a characteristic shared in varying degrees with all information processing systems, is its limited capacity to handle classes of high cardinality, that is, classes having a large number of members. Consequently, when we are presented with a class of very high cardinality, we tend to group its elements together into subclasses in such a way as to reduce the complexity of the information processing task involved. When a point is reached where the cardinality of the class of subclasses exceeds the information handling capacity of the human brain, the boundaries of the subclasses are forced to become imprecise and fuzziness becomes a manifestation of this imprecision. This is the reason why the limited vocabulary we have for the description of colors makes it necessary that the names of colors such as red, green blue, purple, etc., be, in effect, names of fuzzy rather than non-fuzzy sets. This is also why natural languages, which are much higher in level than programming language, are fuzzy whereas programming languages are not.

Fuzziness, then, is a concomitant of complexity. This implies that as the complexity of a task, or of a system for performing that task, exceeds a certain threshold, the system must necessarily become fuzzy in nature. Thus, with the rapid increase in the complexity of the information processing tasks which the computers are called upon to perform, we are reach- ing a point where computers will have to be designed for processing of information in fuzzy form. In fact, it is the capability to manipulate fuzzy concepts that distinguishes human intelligence from the machine intelligence of current generation computers. Without such capability we cannot build machines that can summarize written text, translate well from one natural language to another, or perform many other tasks that humans can do with ease because of their ability to manipulate fuzzy concepts.

It should be noted that the door to the development of fuzzy computers was opened in 1985 by the design of the first fuzzy logic chip by Togai and Watanabe at Bell Telephone Laboratories. The conception and design of a fuzzy computer is one of the basic research projects at LIFE, as well as in Professor Yamakawa's laboratory at Kyushu Institute of Technology. Such a computer may well become a reality during the coming decade. It should be stressed, however, that fuzzy computers will employ both fuzzy hardware and fuzzy software to process fuzzy information. In this and other respects they will be much closer in structure to the human brain than the present-day computers are.

To be able to say more about fuzzy logic and its applications, I will have to clarify some of the issues underlying the concept of a fuzzy set. For this purpose, let us consider a concept, say kind person, which does not admit of a precise definition in terms of a collection of necessary and sufficient conditions. Such a concept may be defined extensionally by associating with each individual who is a member of a universe of discussion $U$, his or her grade of member- 
ship in the fuzzy set of kind persons.

For example, the grade of membership of Robert might be 0.6 , while that of Carol might be 0.9 . These numbers would be elicited from an observer by asking the question "On the scale from 0 to 1 , to what degree is Carol kind?" What is important to note is that the numbers in question are subjective in nature. In the case of Carol, for example, the number 0.9 would not be interpreted as a measure of consensus, that is, as the proportion of individuals who when asked if Carol is kind would respond in the affirmative; nor should 0.9 be interpreted as the probability or the belief that Carol is kind. In this connection, it should be noted that, in general, it is much easier for us to estimate numerical grades of membership than to estimate numerical probabilities.

In the case of a quantifiable concept, the membership function may be represented as a function of one or more measurable attributes. For example, in the case of a young person, the attribute in question would be Age. In this case, the universe of discourse may be taken to be the interval $[0,120]$, and the grade of membership in the class of young people of a person who is, say, 85 years old might be 0.6 .

Clearly, the concepts kind person and young person are both context-dependent and subjective. Some concepts are more contextdependent than others. For example, many is more context-dependent than several, and small is more context-dependent than circular. As will be seen at a later point, in the theory of fuzzy sets, context-dependence plays a key role in the concept of the so-called linguistic variable.
It should be noted that the grade of membership may be interpreted as the truth value of a predicate in multivalued logic. For example, the grade of membership of Carol in the class of kind persons may be equated to the truth value of the proposition "Carol is kind." However, the development of the theory of fuzzy sets and fuzzy logic has followed a path quite different from that of multivalued logic. As an illustration, in the context of the theory of fuzzy sets it is natural to ask the question "What is a convex fuzzy set, or what is meant by the convex hull of a fuzzy set?" This would not be a natural question in multivalued logic. In a more general setting, the theory of fuzzy sets has stimulated the development of a new branch of topology called fuzzy topology, which is a sophisticated mathematical theory in the classical tradition. The conceptual framework of fuzzy topology is related to that of the theory of fuzzy sets but not to multivalued logic.

So far as fuzzy logic is concerned, it may be viewed in two different perspectives: (a) as a branch of the theory of fuzzy sets; and (b) as a generalization of multivalued logic and, especially, as a generalization of Lukasiewicz's L Aleph1 logic. Regardless of the perspective in which it is viewed, fuzzy logic is substantially different in both spirit and detail from the traditional logical systems and provides a much more general framework for knowledge representation and inference.

Among the features of fuzzy logic which are not possessed by traditional logical systems are the following:

(a) In fuzzy logic the truth values are allowed 
to be fuzzy sets labeled true, quite true, very true, more or less true, mostly true, etc. Thus, the truth value of a proposition such as "The crime rate in Japan is very low, may be very true, with very true representing a fuzzy subset of the unit interval.

(b) Classical logical systems, including multivalued logic, allow the use of only two quantifiers, for all (universal quantifier) and there exists (existential quantifier). In fuzzy logic, the quantifiers such as most, many, few, several, usually, etc. are interpreted as fuzzy numbers which serve to describe the absolute or relative cardinalities of fuzzy sets. The importance of this interpretation derives from the fact that, through the use of fuzzy arithmetic, fuzzy logic provides a computational framework for representing the meaning of propositions in a natural language which contain fuzzy quantifiers - as most propositions in a natural language do. This capability of fuzzy logic plays a particularly important role in the representation and inference from imprecise facts and rules in knowledge-based systems.

(c) From the frequentist point of view, fuzzy quantifiers bear a close relation to fuzzy probabilities, e.g., likely, not very likely, unlikely, etc., which underlie much of the commonsense reasoning which we employ in our daily decision-making. Through the connection between fuzzy quantifiers and fuzzy probabilities, fuzzy logic provides a machinery for qualitative decision analysis in which fuzzy utilities are computed from fuzzy probabilities and fuzzy payoff func- tions. Once the fuzzy utilities are known, techniques for the ranking of fuzzy numbers may be employed to determine an optimal course of action.

(d) The conventional approaches to meaning representation in natural languages are based for the most part on predicate logic and its variants. Such approaches do not address an important issue, namely the role played by hedges, e.g., very, more or less, quite, somewhat, extremely, etc. By contrast, fuzzy logic provides a mechanism for dealing with such hedges by interpreting them as operators which act on fuzzy predicates. For example, to a first approximation, the hedge very may be assumed to act as a squaring operator, meaning that the membership function of very tall is the square of the membership function of tall.

As a consequence of having the capability to deal with (a) fuzzy predicates, (b) fuzzy truth values; (c) fuzzy quantifiers; (d) fuzzy probabilities; and (e) hedges, fuzzy logic has a far greater expressive power than traditional logical systems. What this means is that if we were to pick at random a sentence in a book or a newspaper, it would be very unlikely that its meaning could be represented in first-order predicate logic, since a typical sentence in a natural language contains one or more words which are labels of fuzzy predicates. For example, in the sentence It is very unlikely that there will be a significant increase in unemployment in the near future, very unlikely is a fuzzy probability, significant increase is a fuzzy predicate, and so is in the near future. The presence of these predicates presents an obstacle in 
the way of application of first-order predicate logic. By contrast, it is a simple matter to represent the meaning of propositions of this type in fuzzy logic by employing the techniques provided by so-called test-score semantics. In this semantics, the meaning of a proposition is expressed as a procedure which tests the elastic constraints induced by the fuzzy predicates and computes the degree of compatibility of a proposition with an explanatory database. Once the meaning of a proposition is expressed in this manner, the inference techniques of fuzzy logic may be employed to reduce the problem of deduction of an answer to a query to the solution of a mathematical program.

By providing a body of computationallyoriented concepts and techniques for dealing with uncertainty and imprecision, fuzzy logic comes much closer to serving as a descriptive model of human reasoning than traditional logical systems. In this way, fuzzy logic opens the door to many applications which fall beyond the reach of conventional logic-based methods as well as the methods based on classical probability theory. I will not attempt to be more specific at this point by listing the many important applications of fuzzy logic which have been made over the past few years, especially in Japan. It will suffice to mention the highly successful subway system in the city of Sendai which started operating in July, 1987, and has received world-wide recognition. At this juncture, the number of applications of fuzzy logic is growing rapidly in number and variety, with the Japanese scientists and engineers, supported by the government and industry, playing a leading role in the development of a wideranging technological and scientific base for future applications.

Most of the applications of fuzzy logic involve three basic ideas which were put forth in my 1973 paper, "Outline of a New Approach to the Analysis of Complex Systems and Decision Processes."

The first idea relates to the concept of a linguistic variable. A linguistic variable, as its name suggests, is a variable whose values are words or sentences in a natural or synthetic language. For example, if the variable height is treated as a linguistic variable, its values might be expressible as tall, not tall, somewhat tall, very tall, not very tall, very very tall, tall but not very tall, quite tall, more or less tall. Thus, the values in question are sentences formed from the label tall, the negation not, the connectives and and but, and the hedges very, somewhat, quite, and more or less. The motivation for the use of linguistic variables rests on what might be called the principle of minimum specificity. The basic idea underlying this principle is that precision and hence specificity carry a cost and thus should not be used unnecessarily. For example, if I were asked "What is Mary's age?" normally I would not respond by saying twenty three years and five months and five days because such a degree of specificity would not serve a useful purpose. Typically, my response might be young or very young, if the specificity of these terms would be sufficient for the purpose. The guiding principle, then, is do not be more specific than is necessary. This is the essence of the principle of minimum specificity. 
Viewed in this perspective, the basic idea behind the concept of a linguistic variable is that, although linguistic values are less specific than numerical values, they are in many instances sufficiently specific for the purpose on hand.

As a very simple illustration, suppose that we wish to describe in approximate terms the relation between two linguistic variables, Age, which can take the values young, middle-aged, and old, and Color-of-hair, which can take the values black, gray and white. The relation in question may be expressed as three if-then rules:

If Age is young then Color-of-hair is black. If Age is middle-aged then Color-of-hair is gray.

If Age is old then Color-of-hair is white.

Fuzzy if-then rules of this type were employed by Mamdani and Assilian in 1974 in their seminal work on the fuzzy control of a steam engine. In the intervening years, fuzzy logic control has been employed with considerable success in a wide variety of systems ranging from the Sendai subway to Sugeno's fuzzy car and Yamaichi's fund management system. We can expect the application of fuzzy logic control to be greatly accelerated by the availability of fuzzy logic chips which are currently in the initial stages of production and testing. These chips are the descendants of the fuzzy logic chips conceived by Togai and Watanabe in 1985 .

The second basic idea is that of a canonical form. Basically, the concept of a canonical form serves to express a proposition in a natu- ral language as an elastic constraint on a variable. For example, in the case of the proposition Mary is young, the constrained variable is Mary's age, written as Age (Mary), and the elastic constraint on the variable is defined by the fuzzy predicate young. In its canonical form, then, the proposition Mary is young would be expressed as Age (Mary) is young, which places in evidence that the meaning of the proposition in question may be represented as an elastic constraint, young, with the constrained variable being Age(Mary). In this way, a collection of propositions may be expressed as a system of elastic constraints, and the deduction of an answer to a query reduces to the solution of a nonlinear program.

In addition to providing a system for representing the meaning of propositions in a natural language, the concept of a canonical form in combination with the concept of a linguistic variable serves to provide a basis for the representation of qualitative dependencies in systems such as economic systems and complex control systems, which are not sufficiently well defined to admit of analysis by conventional methods. As was alluded to already, many of the practical applications of fuzzy logic make use of a qualitative characterization of input-output relations in the form of fuzzy if-then rules in which the antecedent and consequent propositions are expressed in canonical form.

The third basic idea in fuzzy logic is that of interpolative reasoning. As its name suggests, interpolative reasoning is concerned with a mode of reasoning in which the available information is incomplete, so that an interpolation 
of the available data is required to arrive at an approximate answer to a query. A special case of interpolative reasoning is analogical reasoning, in which the concept of similarity plays a central role. In interpolative reasoning, a comparable role is played by the degree of match between fuzzy predicates.

As a simple illustration of interpolative reasoning, assume that we have two variables $X$ and $Y$ and we know that when $X$ is small $Y$ is large, and $Y$ is small when $X$ is large. The question is: What is the value of $Y$ when $X$ is, say, medium?

The inference rules of fuzzy logic provide a method for computing approximate answers to questions of this type. I cannot go into the technical details of the method in question, but it will suffice to observe that it plays a key role in fuzzy logic control, especially in industrial applications. In such applications, as well as in the realm of expert systems, the importance of interpolative reasoning derives from the fact that the ability to interpolate greatly reduces the need for a large number of if-then rules in the knowledge-base. In this connection, it is of interest to observe that much of human reasoning is interpolative in nature, and that the ability to interpolate depends in an essential way on the remarkable human ability to manipulate fuzzy concepts without a conscious use of the underlying mathematical structure.
I should like to conclude my talk with an optimistic forecast. The past decade has witnessed a rapid growth in the industrial applications of fuzzy logic, especially in Japan. Most of these applications involve the replacement of a skilled human operator by a fuzzy rule-based system. The coming decade will witness a maturing of fuzzy logic and the development of fuzzy computers which will make it possible to replace not just skilled operators but, more importantly, experienced experts. We are beginning to see such applications in the realms of medicine, securities transactions and computer-aided design. And we are likely to see important applications involving a symbiosis of fuzzy logic and neural networks. This may well prove to be a highly promising direction for future research.

Finally, a word of caution. Although fuzzy logic has a much longer reach than traditional logical systems, it has its limitations. There are and will be many tasks which humans can perform with ease and which lie beyond the capability of any computer, any machine and any logical system that we can conceive of today. A simple example of such a task is that of summarizing a non-stereotypical story or a book. At this juncture, we cannot see even dimly the structure of a logic that could be used to perform tasks of this type.

(1989 年 12 月 28 日受付) 


\section{Biographical Note}

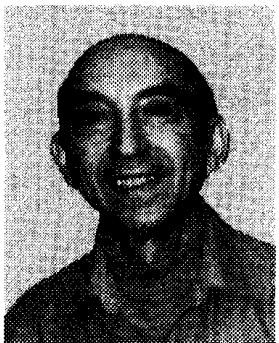

Lotfi A. Zadeh joined the Department of Electrical Engineering at the University of California, Berkeley, in 1959, and served as its chairman from 1963 to 1968. Earlier, he was a member of the electrical engineering faculty at Columbia University. In 1956, he was a visiting member of the Institute for Advanced Study in Princeton, New Jersey. In addition, he held a number of other visiting appointments, among them a visiting professorship in Electrical Engineering at MIT in 1962 and 1968 ; a visiting scientist appointment at IBM Research Laboratory, San Jose, CA, in 1968, 1973, and 1977; and visiting scholar appointments at the AI Center, SRI International, in 1981, and at the Center for the Study of Language and Information, Stanford University, in 1987-1988.

Until 1965, Dr. Zadeh's work has been centered on system theory and decision analysis. Since then, his research interests have shifted to the theory of fuzzy sets and its applications to artificial intelligence, linguistics, logic, decision analysis, expert systems, and neural networks.

An alumnus of the University of Teheran, MIT, and Columbia University, Zadeh is a fellow of the IEEE and AAAS, and a member of the National Academy of Engineering. He was the recipient of the IEEE Education Medal in 1973 and a centennial medal in 1984. Dr. Zadeh was awarded honorary doctorates by the Paul-Sabatier University, France, and the State University of New York, Binghamton, in recognition of his development of the theory of fuzzy sets. In 1989, he was awarded the Honda Prize by the Honda Foundation.

Dr. Zadeh's address is: Computer Science Division, Department of EECS, University of California, Berkeley, CA 94720 , USA. 